\title{
Sparse representation of natural image based on Contourlet overcomplete dictionary
}

\author{
Zhengfang Deng ${ }^{1, a}$, Jing Jin ${ }^{2, b}$, Jinshan $\mathrm{Su}^{1, \mathrm{c}^{*}}$, Xingyu Yang ${ }^{1, \mathrm{~d}}$ \\ ${ }^{1 *}$ College of Electronic and Information, Yili Normal University, Yining, 835000, China \\ ${ }^{2}$ School of Electronic Science and Engineering, Nanjing University, Nanjing, 210046, China \\ aemail: moke8@163.com, bemail: janeking1015@gmail.com, ${ }^{{ }^{\star}}{ }^{\star}$ email: \\ sqsjs1968@aliyun.com, 'email: yangxingyunju@126.com
}

Keywords: Overcomplete Dictionary; Contourlet Basis; Nonlinear Approximation

\begin{abstract}
In this paper, textural features of natural image are researched from the perspective of highly nonlinear approximation theory, in view of the characteristic that a contour of natural image is composed of piecewise regular geometrical curves of the image plane. According to nonlinear approximation theory and multi-scale geometric analysis method, a dictionary based on Contourlet basis function is also proposed in this paper. This dictionary approximates texture area of image by using Orthogonal Matching Pursuit (OMP) method. Experimental results show that Peak Signal to Noise Ratio (PSNR) and Sparsity Ratio (SR) of nonlinear approximated images can be improved effectively by using the proposed dictionary.
\end{abstract}

\section{Introduction}

In the fields of signal and image processing, pattern recognition and computer vision, highly nonlinear sparse representation of image is a basic and core problem. As an important research content of image processing, image approximation is attracting the attention of many researchers and has obtained significant achievements in recent years.

For traditional image sparse representation, most dictionaries are based on Wavelet basis function, and the quality of sparse representation is determined by characteristics of Wavelet. During sparse representation, Wavelet basis function can deal with "point singularity" function effectively [1] [2]. However, it can not obtain optimal nonlinear approximation for the function which is "line singularity". A Wavelet dictionary is the linear combination of functions which are selected from a set of Wavelet basis functions according to certain rules, and used to represent and approximate a signal. Therefore, the Wavelet dictionary can not represent images optimally. In order to describe direction information of two-dimensional function with "line singularity", many multi-scale geometric analysis methods are proposed. Such as Ridgelet transform [2] [3] and Curvelet transform [4] which are proposed by D.L.Donoho, Bandelet transform [5] proposed by E.LePennec and S.Mallat, Contourlets transform [6] and improved Contourlets transform [7] proposed by M.N.Do and M.Vetterli. These new methods have better nonlinear approximation ability than Wavelet in theory. However, a basis function dictionary which is composed of Wavelet and Ridgelet [8] can only deal with "point singularity" and "straight line singularity" effectively but not good at curve singularity of the natural image. As for the construction of dictionary, K-SVD is a popular method [9] which is based on machine learning. It can keep the completeness of sets and search the appropriate dictionary and representing method at the same time. However, its major disadvantage is that this method should adjust the classifications of samples and calculate the adjusted center of cluster continually. When the data is large enough, the time consumption is also large. Therefore, K-SVD can not obtain the optimal approximation effect for the complex natural images.

The characteristics of Contourlet transform are not only multi-resolution and time-frequency locality of time and frequency domains like Wavelet, but also strong directionality and anisotropy. Contourlet transform combines straight line features of multi-scale Ridgelet and point features of Wavelet, and also takes full advantage of multi-scale analysis to obtain sparse representation of 
smooth and edge areas. It can get better effect when dealing with the edge areas and keep detail information of the image.

From the perspective of highly nonlinear approximation theory, a suitable Contourlet overcomplete dictionary aiming at high-dimensional functions with a variety of features is constructed according to the characteristics of Contourlet basis function in this paper. Meanwhile, combined with the Orthogonal Matching Pursuit (OMP) [10] sparse representation algorithm, a new model of complex natural image sparse representation is established. Comparing with multi-scale Ridgelet dictionary [11] and Wavelet dictionary [12] [13], the proposed dictionary has better representation in the aspect of curve singularity, and can improve Peak Signal to Noise Ratio (PSNR) and Sparsity Ratio (SR) of image significantly. Moreover, it has little limit and can represent the most of natural images.

\section{Contourlet Transform and Basis Function}

As shown in Figure 1 (a), it is the process of two-dimensional Wavelet approximating curve singular of a two-dimensional signal. The support area of two-dimensional Wavelet is a square. The higher the resolution is, the smaller the square is. At the same time, the number of Wavelet nonzero coefficients becomes exponential growth and has the performance that it can not represents the original two-dimensional signal "sparsely".

The "strip property" of the geometric regularity is used as a support interval of the basis function, in order to approximate singular curves by the minimum coefficients. Contourlet transform is one of these multi-scale geometric analysis methods. The process of approximating a singular curve by Contourlet is shown in Figure 1 (b).

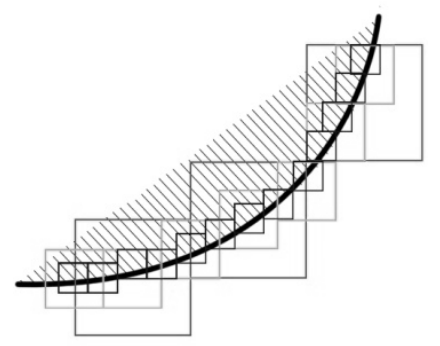

(a) A curve represented by Wavelet transform

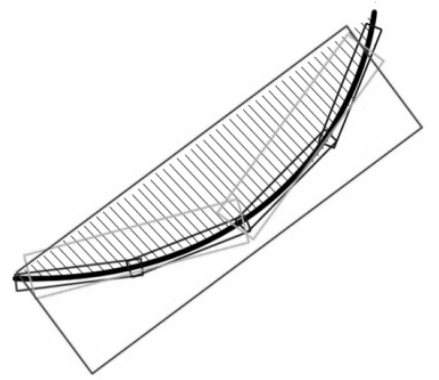

(b) A curve represented by Contourlet transform Fig.1. The approximation of singular curve

The filter bank structured by Contourlet transform is shown in Figure 2. When the low pass filter and directional filter of this structure are orthogonal and completely reconstructed, its discrete transform can achieve perfect reconstruction and has a redundancy less than 3/4.

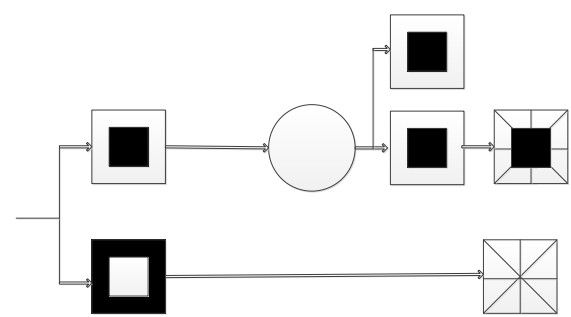

(a) Filter bank structured by Contourlet

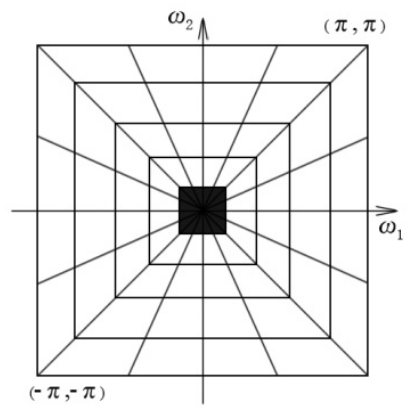

(b) Division of frequency domain of Contourlet

Fig.2. The principle of Contourlet transform.

Considering the acquisition of filter system function, the unit impulse signal is $\delta(\mathrm{j}, \mathrm{k})$ and the system function is $g(j, k)$. The unit impulse response $y(\mathrm{j}, \mathrm{k})$ is: 


$$
\begin{aligned}
y(\mathrm{j}, \mathrm{k}) & =\delta(j, k) * g(j, k) \\
& =\int_{-\infty}^{+\infty} \int_{-\infty}^{+\infty} \delta(\mathrm{m}, \mathrm{n}) g(m-j, \mathrm{n}-k) d m d n,
\end{aligned}
$$

and its Fourier transform result is:

$Y(p, q)=G(p, q)$.

Where $Y(p, q)$ and $G(p, q)$ are Fourier transform of $y(j, k)$ and $g(j, k)$ respectively. It is shown that the unit impulse response $Y(p, q)$ is as same as the system transfer function $G(p, q)$. Therefore, a two-dimensional Contourlet basis function $g(j, k)$ can be calculated by the inverse transformation of $Y(p, q)$.

\section{Establishment of Overcomplete Dictionary and OMP Algorithm}

There are two main problems of image sparse representation based on over complete dictionary. One is how to select the dictionary which can represent the characteristics of image the best. The other one is how to choose the sparse representation algorithm which can represent the image effective and fast.

Considering the signal in space $R^{n}$, the elements in the dictionary are served as columns of matrix and form a matrix $\Psi$ whose size is $n \times p$. Therefore, a signal $x \in R^{n}$ can be expressed as a linear combination of the elements in $\Psi$. When $n<p$, the number of atoms which are used for representing a signal is larger than the dimension of the signal. In this case, the number of columns is greater than rows and the dictionary $\Psi$ is called as a "over-complete dictionary".

Given an over-complete dictionary matrix $D \in R^{n^{*} k}$. Each column of it represents an atom of the original signal. A given signal $y$ can be expressed as a sparse linear combination of these atoms, denoted by $y=D x$ or $y \approx D x$, satisfying $\square y-D x \square_{p \leq \varepsilon}$. The over-completeness of dictionary reflects in that the number of atoms is far greater than the length of signal $y$, that is $n \square k$.

Given a set $D=\left\{g_{k}, \mathrm{k}=1,2, \ldots \mathrm{K}\right\}$, and its elements are unit vectors of $H=R^{N}$ in whole Hibert space, where $K \geq N$. The set $D$ is called as an atomic library, which is also called as a dictionary. For any signal $f \in H$ in this space, it is can be decomposed by selecting $l$ atoms adaptively in the library:

$$
f_{1}=\sum_{\gamma \in I_{l}} c_{\gamma} g_{\gamma} .
$$

Where, $I_{l}$ is the subscript of $g_{r}$, and $l$ is sparsity degree. The optimal subset composed of $l$ atoms in $D$ is:

$$
B=\operatorname{span}\left(\mathrm{g}_{k}, \gamma \in \mathrm{I}_{l}\right) \text {. }
$$

The approximation error is defined as:

$$
\sigma_{l}(f, f D)=\underset{f_{l}}{\inf }\left\|f-f_{l}\right\|
$$

OMP algorithm is proposed by Pati and Davis firstly [14]. Its decomposition steps are as follows:

(1) Calculating the inner product of the signal $y$ and atoms of each column in dictionary. The atom whose absolute value is maximum is selected as the best match atom of $y$ in this iteration, named as $x_{r_{0}}$. It satisfies $\left|<y, x_{r_{0}}>\right|=\sup _{i \in(1, \ldots k)}\left|<y, x_{i}>\right|$, where $r_{0}$ represents an index of columns of the dictionary matrix. Therefore, $y$ can be decomposed into the vertical projection of $x_{r_{0}}$ and residual value, that is $y=<y, x_{r 0}>x_{r 0}+R_{1} f$.

(2) Decomposing the residual value by using the same step above. $R_{k} f=<R_{k} f, x_{r k+1}>+R_{k+1} f$ can be obtained in step $k$, where $x_{r k+1}$ satisfies $\left|<R_{k} f, x_{r k+1}>\right|=\sup _{i \in(1, \ldots k)}\left|R_{k} f, x_{i}\right|$. It is shown that 
the signal $y$ is decomposed as $y=\sum_{n=0}^{k}<R_{n} f, x_{r n}>R_{n} f+R_{k+1} f$ after $k$ steps, where $R_{0} f=y$.

\section{Experimental Results}

In the experiments, standard images and natural photos are nonlinear approximated by Wavelet Basis Dictionary (WBD) and Contourlet Basis Dictionary (CBD) separately, and Sparsity Ratio (SR) of each method are compared.

Approximation of "Cameraman" which is $256 \times 256$ is shown in Fig. 3. The original image as shown in Figure 3 (a) is approximated using OMP algorithm, and the number of Iteration is $k=200$. The size of dictionary is $256 \times 1024$ which means it contains 1024 atoms. The approximation results by WBD and CBD are shown in Figure 3 (b) and (c). It is shown that the sparse representation by WBD is not good enough and has lower PSNR, because Wavelet is not good at two-dimensional image which has "line singularity". While CBD can't only deal with both "point singularity" and "straight singularity", but also represent "curve singularity" of image. PSNR of CBD is also improved obviously. Local enlarged images are shown in Figure 3 (d) to (f). It is obviously shown that the dictionary proposed in this paper has better approximation effect and less noise than WBD.

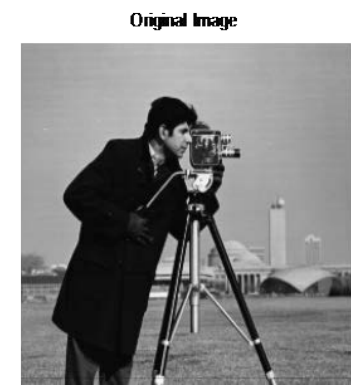

(a) Original Cameraman Oniginal mage

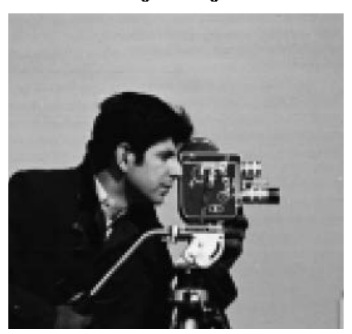

(d) Local enlarged of (a)
Wawekt Spasily mage

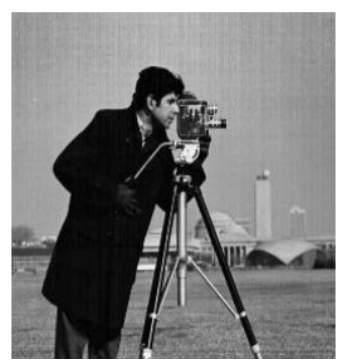

(b) Approximation image by WBD Wavetet Sparsity mage

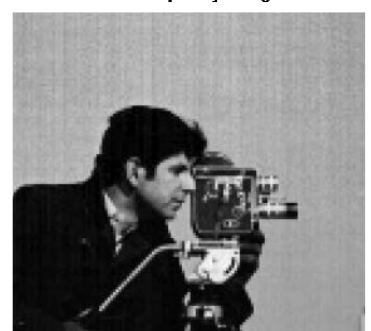

(e) Local enlarged of (b)

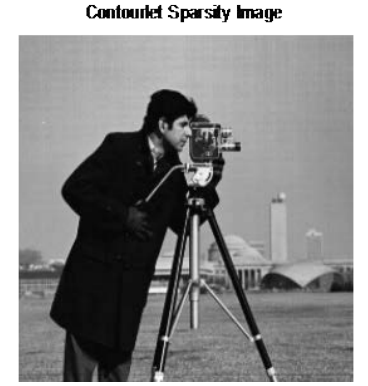

(c) approximation image by CBD Coritount Sparsity mage

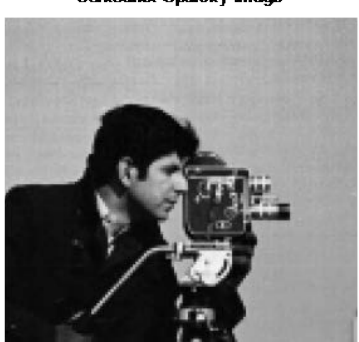

(f) Local enlarged of (c)

Fig.3. Approximation results of Cameraman.

Sparsity Ratio (SR) is defined as $S R=\frac{\text { The total number of pixels of the image }}{\text { The total number of image coefficients }}$.

It has a certain corresponding relationship with threshold value (THR). That is the sum of all pixels which represent coefficients of image is the total number of the pixels which are larger than THR in the image processed by Wavelet or Contourlet Transform. For example, the relationship of a $256 \times 256$ image is shown in Figure 4 .

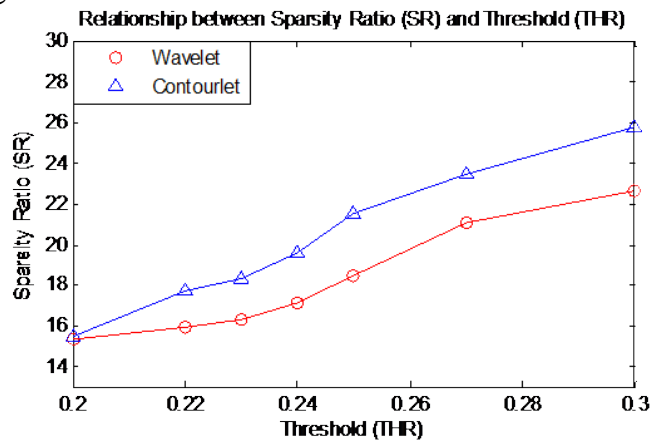

Fig.4. The relationship between SR and THR. 
Moreover, CBD is robust for natural photos. Experimental result is shown in Figure 5. It is shown that the dictionary proposed has better approximation result than WBD with the same THR. While, by using the same CBD, the smaller the THR is, the fewer pixels are used for sparse representation and the smaller the PSNR is. Therefore, the quality of approximated image is better.

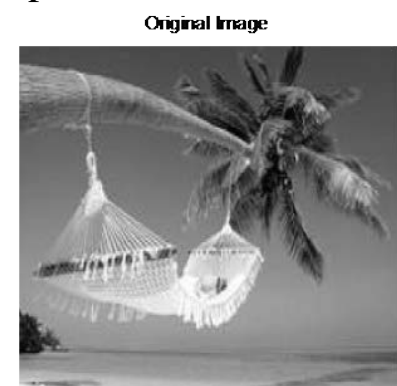

(a) Original image

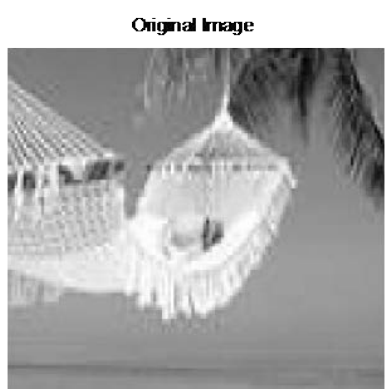

(d) Local image of (a)

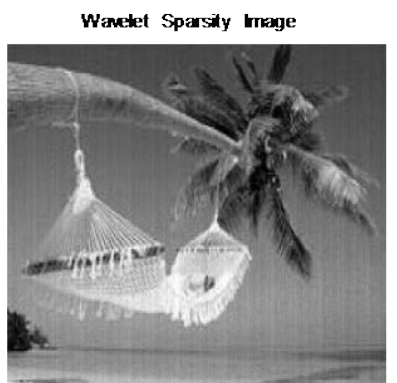

(b) WBD (THR=0.22)

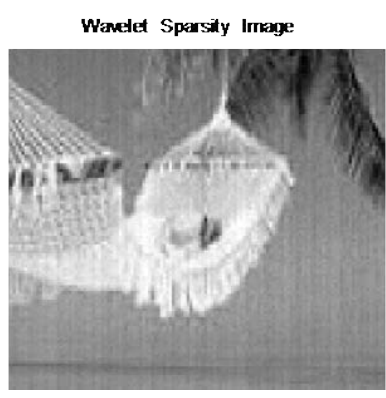

(e) Local image of (b)

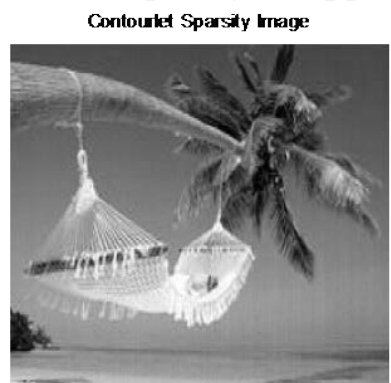

(c) CBD (THR=0.22)

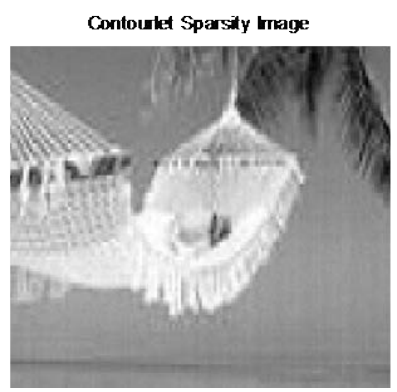

(f) Local image of (c)

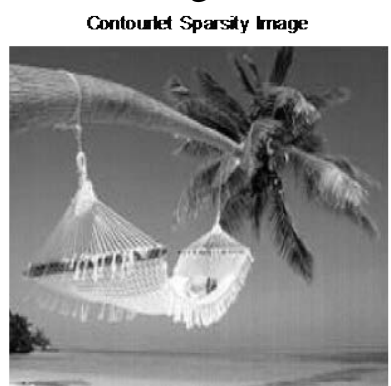

(d) CBD (THR=0.235)

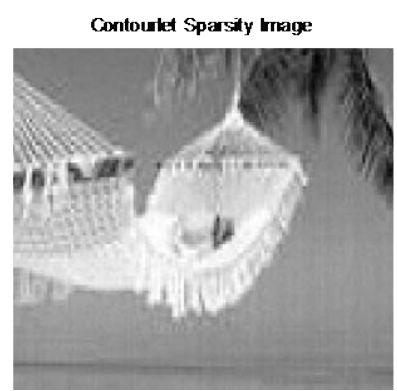

(g) Local image of (d) Fig.5. Approximation results of Beach.

PSNR with different THR of some images are shown in Table 1. It is shown that the proposed dictionary has better PSNR obviously than WBD in a fixed THR.

Table 1. PSNR of approximated images.

\begin{tabular}{cccc}
\hline Images & $\begin{array}{c}\text { Threshold } \\
\text { (THR) }\end{array}$ & PSNR of WBD & PSNR of CBD \\
\hline Cameraman & 0.22 & 39.9203 & 41.4108 \\
& 0.235 & 37.9334 & 41.6363 \\
Beach & 0.25 & 34.6432 & 37.4152 \\
& 0.22 & 37.8103 & 40.2479 \\
Lena & 0.235 & 36.8164 & 40.2234 \\
& 0.25 & 32.7244 & 36.3537 \\
\multirow{2}{*}{ Boat } & 0.22 & 38.8467 & 40.9560 \\
& 0.235 & 37.5695 & 30.6638 \\
\multirow{2}{*}{ Waterfall } & 0.25 & 33.9672 & 39.2062 \\
& 0.22 & 37.4578 & 39.8979 \\
& 0.235 & 36.3471 & 36.13258 \\
& 0.25 & 32.4843 & 41.7236 \\
& 0.22 & 38.6981 & 41.4988 \\
\end{tabular}

\section{Conclusion}

Contourlet transform provides a flexible and multi-resolution orientation decomposition method of the image. It allows different number of directions of decomposition on each scale, and the final approximation result is similar to the basis structure of Contour segment. According to this characteristic, a dictionary based on Contourlet basis function is proposed in this paper. This dictionary can deal with not only "point singularity" and "straight singularity" but also "curve singularity" and has good approximation performance. Through the proposed dictionary combined 
with OMP algorithm, not only in the approximated image obtains better result of nonlinear approximation and has greatly improved sparse representation capability than traditional methods. Because of the advantages above, the proposed dictionary is advantageous to the practical application and next research stage.

\section{Acknowledgement}

In this paper, the research was sponsored by the Educational Commission of Xinjiang Autonomous Region of China (Grant NO. XJEDU2011I49) and the Fundamental Research Funds for Yili Normal University (Grant NO. 2011YNZD011).

\section{References}

[1] GROSSMANN A, MORLET J. Decomposition of Hardy functions into square integrable Wavelets of constant shape [J]. SIAM Journal on Mathematical Analysis, 1984, 15(1): 722-736.

[2] DONOHO D L. Orthonormal Ridgelets and linear singularities [R]. Stanford: Stanford University. Department of Statistics, 1998.

[3] CANDES E J. Monoscale Ridgelets for the Representation of Images with Edges [R]. Stanford: Stanford University. Department of Statistics, 1999.

[4] STARCK Jean-Luc, CANDÈS Emmanuel J, DONOHO D L. The curvelet transform for image denoising [J]. IEEE Trans. on Image Processing, 2002, 11(6): 670-684.

[5] G. Peyre, S. Mallat. Discrete Bandelets with geometric orthogonal filters [C]//Proceedings of IEEE International Conference on Image Processing. Genoa, Italy: IEEE, 2005: 65-68.

[6] DO M N, VETTERLI M. The contourlet transform: an efficient directional multiresolution image representation [J]. IEEE Trans. on Image Proc. 2005, 14(12): 2091-2106.

[7] Su Jinshan, Feng Yan, An Improved contourlet transform and its application in image denosing [J]. Journal of computer aided design\&computer graphics. 2009, 21(11): 1612-1617.

[8] Deng Chengzhi, Image Denoising Algorithm Based on Multiscale Ridgelet Dictionary [J]. Computer Engineering, 2010, 36(23): 207-209.

[9] Ribhu, D.Ghosh, Dictionary Design for Sparse Signal Representations Using K-SVD with Sparse Bayesian Learning [C]. Bei Jing: ICSP 2012 Proceedings, 2012: 21-25.

[10] Xie Qinlan, Ding Jingjing, Image Denoising Method Based on Modified Dictionary Learning Algorithm [J]. Computer \& Digital Engineering, 2014, 06(42): 1071-1074.

[11] Xie Kai, Zhang Fen, Image Denoising method based on dictionary learing with union of two orthonormal bases [J]. Computer application, 2012, 32(4): 1119-1121.

[12] L. Rebollo-Neira, BOWLEY J, Constantinidesb, PLASTINOC A. Self Contained Encrypted Image Folding [J]. Physica A: Statistical Mechanics and its Applications, 2012, 391(23): 5858-5870.

[13] Tian Liping, Wang Jianguo, Target Recognition of SAR Images Based on Sparse Representation of Wavelet Dictionary [J]. Radar Science and Technology, 2014, 1(12): 45-51.

[14] MALLAT S, ZHANG Z. Matching pursuits with time-frequency dictionaries [J]. IEEE Trans. on Signal Processing, 1993, 41(12): 3397-3415. 